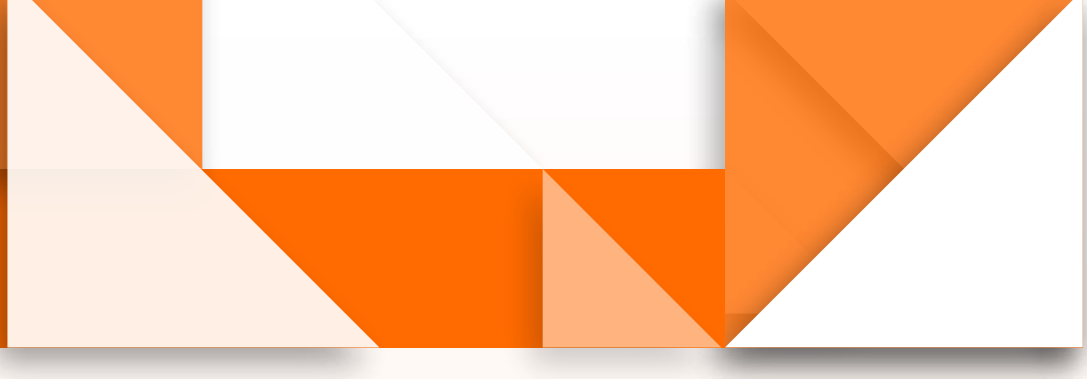

\title{
Investigaciones en Colombia sobre libros de texto de química: análisis documental
}

\section{- Research in Colombia on Chemistry Textbooks: Documentary Analysis}

- Pesquisas na Colômbia sobre livros de texto de química: análise documental

\section{Resumen}

Los libros de texto han sido investigados desde diversas perspectivas, sobre todo desde aportes de la didáctica de las ciencias. Considerando esto, se propuso, mediante un enfoque cualitativo con análisis de contenido, caracterizar tendencias de investigación en libros de texto de química (LTQ) en Colombia. Así, se analizaron 33 documentos (disertaciones, artículos y ponencias) que investigaron sobre este tema. Las categorías encontradas evidencian una tendencia de investigación sobre conceptos químicos, análisis histórico-epistemológicos, pesquisas sobre errores conceptuales, imágenes distorsionadas de la química, falta de relación entre lo metadisciplinar e interdisciplinar y un constructivismo incipiente.

Palabras clave

libros de texto; química; contenidos; educación científica

\begin{abstract}
Textbooks have been investigated from perspectives such as science education, which have contributed results for its improvement. Considering this, we decided to characterize the research trends regarding chemistry textbooks (Ст) in Colombia through a qualitative approach with content analysis. Accordingly, we analyzed 33 documents (dissertations, articles, and lectures) that researched this topic and which made it possible to identify the emergence of 4 categories (content analysis, didactic analysis, evolution of $\mathrm{CT}$, and the authors) that show a tendency of research on chemical concepts, historical-epistemological analysis, research on conceptual errors, distorted images of chemistry, lack of relationship between that which is metadisciplinary and interdisciplinary, and an incipient constructivism.
\end{abstract}

Keywords

textbooks; chemistry; content; scientific education

\section{Diana Lineth Parga Lozano*}

Magíster en Docencia de la química y profesora del Departamento de Química de la Universidad Pedagógica Nacional.

dparga@pedagogica.edu.co.

ORCID: http://orcid.org0000-0002-7899-0767.

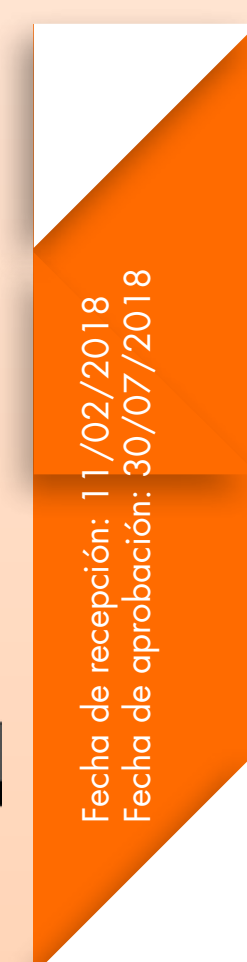




\section{Resumo}

Os livros de texto têm sido pesquisados desde perspectivas como a didática das ciências, que contribuíram com resultados para seu melhoramento. Considerando isso, proponemos mediante uma abordagem qualitativa com análise de conteúdo, caracterizar tendências de pesquisa em livros de texto de química (LTQ) na Colômbia. Assim, analisamos 33 documentos (dissertações, artigos e ponencias) que investigaram sobre este tema e permitiram identificar o surgimento de 4 categorias (análise de conteúdos, análise didático, evolução dos LTQ e os autores) que evidenciam uma tendência de pesquisa sobre conceitos, análises histórico-epistemológicas, pesquisas sobre erros conceituais, imagens distorsionadas da química, falta de relação entre o metadisciplinar e interdisciplinar e um constructivismo incipiente.

Palavras-chave

livros de texto; química; conteúdos; educação científica 


\section{Introducción}

Los libros de texto han sido objeto de investigaciones variadas, lo que los hace claves en la investigación y en la enseñanza de las ciencias. Estos hechos favorecen que la didáctica de la química los asuma como objetos de estudio para su mejor conocimiento, lo cual ha contribuido a la enseñanza-aprendizaje relacionada con una comprensión del conocimiento químico en todas sus dimensiones. Así, Occeli y Valeiras (2013) consideran que la diversidad de estudios genera dispersión de conocimiento sobre las características de estos materiales y su impacto en la enseñanza de las ciencias. Sobre la diversidad de estudios, en el ámbito internacional se encuentra que la tendencia en las investigaciones en libros de texto se centra en analizar contenidos de la química (cuántica, teorías, estructura de la materia y modelos atómicos (Farias, 2012; González-Canle y Sánchez-Gómez, 2014; Malaver, Pujol d'Alessandro, 2004); estudios relacionados con la historia al abordar conceptos particulares y el papel de las ilustraciones (Perales y Vílchez, 2015) y de las actividades de aprendizaje (López-Valentín y Guerra-Ramos, 2013). Para analizar lo que sucede en Colombia, se propuso realizar un estudio documental de las investigaciones sobre libros de texto de química (LTQ), en educación media y universitaria, para compararlas con las categorías del contexto internacional, ver su relación y formas de mejorarlos.

Esto es importante como antecedente y balance del tema investigado en Colombia, porque los LTQ desarrollan en detalle temas desde contenidos, gráficos, imágenes, datos seleccionados y tabulados, ejercicios y problemas, imágenes de la naturaleza del conocimiento científico-tecnológico asociados a la química, la formación en valores y otras características importantes para el estudiantado, para lo cual se estructuran secciones en los LTQ (contenidos, secuenciación, lecturas, evaluaciones, trabajos prácticos de laboratorio, ejercicios, problemas, proyectos, etc.) que llaman la atención del contenido científico, que en muchos casos es indiferente y tedioso para el alumnado. Sin embargo, żson suficientes estas características para los estudiantes, para los ciudadanos que requiere la sociedad? ¿De qué forma los LTQ pueden ayudar en esto? Así, se planteó determinar desde un análisis documental las tendencias de investigación en LTQ en Colombia, considerando el libro de texto como recurso que aporta al proceso de enseñanza-aprendizaje; que integra conocimientos y saberes (pedagógico-didácticos, metadisciplinares, etc.) con las necesidades de los estudiantes a los cuales van dirigidos.

\section{Metodología}

Se hallaron tres tipos de documentos que expresan investigaciones sobre libros de texto de química: trabajos de grado, trabajos de maestría y artículos. A estos se les hizo un estudio cualitativo e interpretativo (Denzin y Lincoln, 2012; Valles, 2009), y se encontró en total 1 trabajo de grado (TG), 16 disertaciones de maestría (DM) y 16 artículos publicados (AP) sobre investigaciones en LTQ en los últimos diez años en Colombia (2016-2007) (véase el anexo 1). Las fases del estudio fueron:

Fase 1: búsqueda, selección y organización de documentos. Contempló revisión de repositorios y bases de datos. Se buscaron términos como libros, texto, química, desde buscadores bibliográficos. Se encontraron 33 trabajos, que constituyen los documentos del análisis o texto de campo (Ruiz, 2009).

Fase 2: caracterización. Se leyó el texto de campo, marcando aspectos relevantes en cuanto a objetivos, metodologías y resultados. Se obtuvo el texto de investigación con notas y 
sistematizaciones, como recomienda Ruiz (2009). Se hicieron categorizaciones, primeras interpretaciones y comparaciones. En este proceso, se usó el análisis de contenido como técnica de análisis de datos.

Fase 3: análisis. Se interpretaron y analizaron las tendencias de investigaciones sobre LTQ en Colombia desde los datos de la fase 2.

Con el análisis de contenido como "procedimiento que desestabiliza la inteligibilidad inmediata de la superficie textual" (Galeano, 2012, p. 126), se definieron como unidades de registro la palabra, la frase y el concepto, que se interrelacionan para construir sistemas de categorías, y abarcar aspectos relevantes de los textos según el objetivo de investigación. Como unidad de contexto (lugares concretos en el texto, que ubica las unidades de registro) se usó el párrafo para localizar y ubicar las unidades de registro, haciendo evidente su significado y condiciones de producción (Galeano, 2012). Con la codificación se identificaron presencias, frecuencias y recurrencias. La categorización se hizo luego de la lectura general de los textos de campo: clasificación semántica para identificar categorías y subcategorías analíticas emergentes (véase la tabla 1).

\section{Tendencias de investigación en libros de texto de química en Colombia}

La metodología usada permitió evidenciar la emergencia de las categorías en las que se investigan LTQ en Colombia en educación media y universitaria; es decir, categorías que resultaron del análisis documental propuesto y que configuran las tendencias de investigación (véase la tabla 1).

\section{Categoría 1: análisis de los contenidos (AC)}

Esta categoría emergió al identificar en los documentos analizados, investigaciones de los contenidos disciplinares (sobre todo conceptos de química) y metadisciplinares.

\section{Subcategoría Contenido disciplinar de química}

Describe contenidos curriculares o el contenido disciplinar (CD) de la química para educación media según el Ministerio de Educación Nacional (MEN, 2004) y universitaria. Si bien predominan estudios de CD de educación media (LTQ-EM), también los hubo de LTQ universitarios (LTQ-U). Estos contenidos son: modelo atómico de Rutherford (Cuéllar, 2004; Cuéllar, Gallego y Pérez, 2008), enlace químico (López, Saldarriaga y Tamayo, 2007), electroquímica (Villa, 2008), isómeros (Gómez-Hernández, 2009), modelo del octeto de Lewis y Langmuir (Herreño, 2009; Herreño, Gallego y Pérez, 2010), modelos moleculares de Walther Kossel, Gilbert Newton Lewis y Linus Carl Pauling (Moreno, 2009; 2016), mol y cantidad de sustancia (García, 2009; García y Parga, 2009), teoría estructural en 
química orgánica (QO) (Jurado, 2009; Parga y Alba, 2015), hipótesis de Avogadro (Farieta, 2010a; 2010b), modelo semicuántico de Bohr (Moreno, Gallego y Pérez, 2010), teorías del oxígeno y del flogisto (Ariza, 2009; Ariza y Parga, 201 1; Parga y Alba, 2015), proteínas (Ahumada, 2011), combustión (Ariza y Parga, 2011; Cabrera, 2012), pH (Alméciga y Muñoz, 2013), discontinuidad de la materia (Gómez-Poveda, 2010; Parga y Alba, 2015), principio de Le Chatelier y equilibrio químico (Pelayo, 2014), aromaticidad (Montaña y Piraban, 2014), hibridación (Burgos y Gómez-Romero, 2014), reacciones químicas (Gutiérrez y Reyes, 2014), fenómeno químico (Garzón y Pérez, 2015) y valencia (Tejada, Acevedo y Gattas, 2015). La tabla 2 resume los principales hallazgos.

La tabla 2 evidencia los contenidos analizados en términos de conceptos, teorías y modelos de la química, predominando estudios sobre los conceptos (véase la tabla 1). Estos estudios plantean que los conceptos están descontextualizados históricamente y desligados de las teorías de la química (descontextualización disciplinar), incluso los documentos analizados enfatizan en tal tipología de contenido. La mayoría de trabajos evidencia errores conceptuales o conceptos que hoy la química no usa.

El $21 \%$ de los trabajos (según la tabla 1) analiza contenidos diferentes al disciplinar o conceptual: contenidos procedimentales y actitudinales. Los contenidos procedimentales se refieren a procesos que debe desarrollar el estudiante, y su evolución y técnicas y estrategias para resolver situaciones propuestas.

Tabla 1. Categorías y subcategorías emergentes en el análisis de investigaciones en LTQ en Colombia

\begin{tabular}{|c|c|c|c|}
\hline Categorías & Subcategorías & Características & $\begin{array}{c}\% \text { de } \\
\text { documentos }\end{array}$ \\
\hline \multirow[t]{2}{*}{$\begin{array}{l}\text { 1. Análisis de los } \\
\text { contenidos (AC) }\end{array}$} & $\begin{array}{l}\text { Contenidos disciplinares } \\
\text { (CD) }\end{array}$ & $\begin{array}{l}\text { Contenidos disciplinares definidos para la } \\
\text { enseñanza de la química. }\end{array}$ & CD: $100 \%$ \\
\hline & $\begin{array}{l}\text { Contenidos } \\
\text { metadisciplinares (CM) }\end{array}$ & $\begin{array}{l}\text { Contenidos con relaciones entre historia y } \\
\text { epistemología (HE), sociología, enfoque ciencia, } \\
\text { tecnología y sociedad (CTS), y contenidos } \\
\text { ambientales o CA. }\end{array}$ & $\begin{array}{l}\text { HE: } 94 \% \\
\text { CTS: } 3 \% \\
\text { CA: } 3 \%\end{array}$ \\
\hline \multirow[t]{3}{*}{ 2. Análisis didáctico O AD } & $\begin{array}{l}\text { Implicaciones didácticas } \\
\text { (ID) }\end{array}$ & $\begin{array}{l}\text { Analiza implicaciones didácticas desde modelos } \\
\text { didácticos expresados en la propuesta editorial. }\end{array}$ & \multirow[t]{3}{*}{$21 \%$} \\
\hline & $\begin{array}{l}\text { Intenciones de enseñanza } \\
\text { (IE) }\end{array}$ & $\begin{array}{l}\text { Objetivos, competencias, actividades*, secciones } \\
\text { y demás criterios del proceso de enseñanza- } \\
\text { aprendizaje. }\end{array}$ & \\
\hline & Formas de evaluar (FE) & Actividades para evaluar el proceso de aprendizaje. & \\
\hline $\begin{array}{l}\text { 3. Evolución (E) de los } \\
\text { LTQ }\end{array}$ & & $\begin{array}{l}\text { Evolución de modelos didácticos, de contenidos y } \\
\text { concepciones de la química. }\end{array}$ & $3 \%$ \\
\hline \multirow{3}{*}{$\begin{array}{l}\text { 4. Los autores (A) } \\
\text { en cuanto a sus } \\
\text { conocimientos/ } \\
\text { creencias }\end{array}$} & Metadisciplinares (M) & $\begin{array}{l}\text { Conocimientos/creencias epistemológicas, } \\
\text { históricas. }\end{array}$ & \multirow[t]{3}{*}{$3 \%$} \\
\hline & $\begin{array}{l}\text { Pedagógico-didácticas } \\
\text { (PD) }\end{array}$ & $\begin{array}{l}\text { Conocimientos/creencias de la didáctica de la } \\
\text { química. }\end{array}$ & \\
\hline & Químicas (Q) & $\begin{array}{l}\text { Conocimientos-creencias de conceptos, principios, } \\
\text { teorías y modelos. }\end{array}$ & \\
\hline
\end{tabular}

Fuente: elaboración propia.

*Clasificadas como contenidos procedimentales y actitudinales 
Tabla 2. Subcategoría del contenido disciplinar (CD) en LTQ

\begin{tabular}{|c|c|c|}
\hline Contenido & CD & Característica del análisis \\
\hline \multirow[t]{12}{*}{ Conceptos } & Electroquímica & $\begin{array}{l}10 \text { LTQ-U. Estos no evalúan ideas previas del concepto ni } \\
\text { se establecen relaciones con lo cotidiano. Hay deficiencias } \\
\text { respecto a la historia del concepto y explicaciones erradas } \\
\text { de la función del puente salino; no se distingue entre celda, } \\
\text { pilas y baterías; hay distorsiones entre ánodo, cátodo, } \\
\text { potencial de electrodo, diferencia de potencial, fuerza } \\
\text { electromotriz, potencial de celda y voltaje de celda. }\end{array}$ \\
\hline & Proteínas & $\begin{array}{l}5 \text { LTQ-EM. Con análisis histórico epistemológico que evidencia } \\
\text { deficiencia frente al trabajo científico. El abordaje didáctico } \\
\text { se centra en transmisión de información en la mayoría de los } \\
\text { textos. }\end{array}$ \\
\hline & Enlace químico & $\begin{array}{l}2 \text { LTQ-EM. Analizan } 37 \text { figuras para representar el concepto. } \\
\text { Su uso es más estético que didáctico. Hay diseños } \\
\text { inadecuados que inducen a errores; falta conexión entre la } \\
\text { figura, el concepto y lo explicado. }\end{array}$ \\
\hline & Isómeros & $\begin{array}{l}4 \text { LTQ-U. Trabajan clasificación de isómeros y su vinculación } \\
\text { histórico-epistemológica; se evidencian descripciones } \\
\text { enciclopédicas, sin los conceptos de estructura molecular. }\end{array}$ \\
\hline & Hipótesis de Avogadro & $\begin{array}{l}14 \text { LTQ-EM y LTQ-U. Analiza la transposición didáctica que } \\
\text { evidencia diferencias que reducen los aportes de Avogadro } \\
\text { y desconocen los aportes de Dalton y Gay Lussac; no } \\
\text { se explica cómo se llegó a esta hipótesis, ni como la } \\
\text { comunidad aporta al validarla en el Congreso de Karlsruhe, } \\
\text { ni su importancia para establecer los pesos atómicos de } \\
\text { Dumas y Cannizzaro. }\end{array}$ \\
\hline & Combustión & $\begin{array}{l}6 \text { LTQ-U. Se analizaron conceptos como combustión, } \\
\text { calcinación, corrosión, respiración, reducción y oxidación. } \\
\text { Las formas de preguntar y los experimentos son } \\
\text { demostrativos. El concepto central está desligado de los } \\
\text { demás y se abordan desde lo micro hacia lo macroscópico. } \\
\text { Lo histórico está ausente. }\end{array}$ \\
\hline & Fenómeno químico & $\begin{array}{l}4 \text { LTQ-EM. Analiza la versión del concepto desde su lenguaje } \\
\text { y el papel explicativo de los modelos, la que se usa como } \\
\text { noción, concepto, categoría o modelo. }\end{array}$ \\
\hline & Hibridación & $\begin{array}{l}11 \text { LTQ-U (del periodo 1980-2006). Se determina la versión } \\
\text { epistemológica del concepto enseñado y su correspondencia } \\
\text { con el modelo de Pauling. }\end{array}$ \\
\hline & Valencia & $\begin{array}{l}2 \text { LTQ-EM y } 9 \text { LTQ-U. Este concepto se aborda de forma } \\
\text { incompleta y estática; no se enseña su evolución histórica. } \\
\text { No hay consenso del uso del concepto: se emplean } \\
\text { expresiones como adjetivo, sustantivo, término, número; no } \\
\text { se diferencia entre valencia primaria (número de oxidación), } \\
\text { ni secundaria (número de coordinación). }\end{array}$ \\
\hline & $\mathrm{pH}$ & $\begin{array}{l}10 \text { LTQ-U. En algunos de estos textos la química es una } \\
\text { ciencia en contexto social pero se asume un enfoque de } \\
\text { conocimiento ahistórico, descontextualizado, acumulativo e } \\
\text { individualista frente a la construcción del concepto. }\end{array}$ \\
\hline & $\begin{array}{l}\text { Principios de Le } \\
\text { Chatelier y equilibrio } \\
\text { químico }\end{array}$ & $\begin{array}{l}25 \text { LTQ-U (del periodo 1979-2012). El concepto no } \\
\text { se corresponde histórica ni epistemológicamente con } \\
\text { lo establecido por las actividades científicas (visión } \\
\text { distorsionada de la ciencia), lo que fomenta reduccionismo } \\
\text { y tergiversaciones en la enseñanza y lleva a la poca } \\
\text { confiabilidad en estos materiales. Lo enseñado corresponde } \\
\text { a las primeras visiones de Le Chatelier. }\end{array}$ \\
\hline & Aromaticidad & $\begin{array}{l}10 \text { LTQ-U. El abordaje del concepto deja de lado aspectos } \\
\text { sobre la aromaticidad, sU entendimiento desde criterios } \\
\text { magnéticos y energéticos (estabilidad de la molécula); los } \\
\text { autores del libro enfatizan en la nomenclatura de aromáticos } \\
\text { y desconocen aspectos HE de su desarrollo. }\end{array}$ \\
\hline
\end{tabular}




\begin{tabular}{|c|c|c|}
\hline Contenido & CD & Característica del análisis \\
\hline & Reacciones químicas & $\begin{array}{l}4 \text { LTQ-U (del periodo 1999-2003). Se presenta de formas } \\
\text { diversas: previo al enlace químico, tabla periódica, } \\
\text { estructura atómica; es un contenido matematizado que } \\
\text { se aborda como ejercicios por resolver; hay una historia } \\
\text { anacrónica; el papel del experimento es demostrativo. }\end{array}$ \\
\hline \multirow[t]{4}{*}{ Modelos } & Modelo atómico & $\begin{array}{l}5 \text { LTQ-EM. Se determina la confiabilidad de los LTQ a partir } \\
\text { del desarrollo HE del modelo presentado por Rutherford y } \\
\text { su comparación con lo abordado por los libros; hay errores } \\
\text { conceptuales que cuestionan la confiabilidad y se aleja de lo } \\
\text { planteado por la química. }\end{array}$ \\
\hline & Modelo del octeto & $\begin{array}{l}5 \text { LTQ-EM. Desde un análisis HE hay falta de referencias a la } \\
\text { construcción del modelo; se alude a la fórmula de Lewis, sin } \\
\text { reconocer los aportes de l. Langmuir. El empleo del modelo } \\
\text { se reduce a representar electrones de valencia. }\end{array}$ \\
\hline & Modelos moleculares & $\begin{array}{l}15 \text { LTQ-EM. Se analizó el proceso de transposición didáctica } \\
\text { respecto a los aportes HE de modelos moleculares; no se } \\
\text { alude a los científicos que propusieron los modelos ni al } \\
\text { modelo original.Los modelos se presentan como ciertos, } \\
\text { únicos, visibles y absolutos. }\end{array}$ \\
\hline & Modelo semicuántico & $\begin{array}{l}7 \text { LTQ-EM (periodo 1984-2005) y } 10 \text { LTQ-U (periodo 1983- } \\
\text { 2007). Se muestra una diferencia entre la propuesta de } \\
\text { Bohr de } 1913 \text { y la transposición del modelo en los LTQ. Se } \\
\text { evidencian modelos elaborados, sin contexto histórico. }\end{array}$ \\
\hline \multirow[t]{4}{*}{ Teorías } & Teoría estructural QO & $\begin{array}{l}4 \text { LTQ-EM (del periodo 1997-2003). Un solo libro presenta } \\
\text { el desarrollo de las teorías de la química orgánica; } \\
\text { los demás solo presenta el tema de fórmulas químicas } \\
\text { descontextualizado de la teoría que las originó. }\end{array}$ \\
\hline & $\begin{array}{l}\text { Teorías del oxígeno y } \\
\text { del flogisto }\end{array}$ & $\begin{array}{l}6 \text { LTQ-EM (periodo 1997-2010). Análisis para comprender } \\
\text { el concepto de combustión. En } 4 \text { LT, el contenido se } \\
\text { centra en el concepto explicativo preferente: definiciones, } \\
\text { datos, hechos dentro del marco de la disciplina química; } \\
\text { se encuentran escasas leyes (conjunto compacto), pocos } \\
\text { conceptos de dominio ordenado, correlación y reglas que } \\
\text { permitan ordenar los elementos que dan sustento a la } \\
\text { estructura de cuerpo teórico del modelo teórico. Los LTQ } \\
\text { manifiestan una concepción hibrida entre flogisto y teoría del } \\
\text { oxígeno. }\end{array}$ \\
\hline & $\begin{array}{l}\text { Equivalentismo } \\
\text { atomismo }\end{array}$ & $\begin{array}{l}8 \text { LTQ-EM (del periodo 1986-2002). Análisis hecho desde } \\
\text { estas teorías y sus conceptos mol y cantidad de sustancia y } \\
\text { desde lo disciplinar, histórico, psicopedagógico y el contexto } \\
\text { escolar. Se hallan visiones equivalentistas (normalidad, } \\
\text { equivalente gramo, átomo gramo, molécula gramo y } \\
\text { peso molecular), macroscópicas y predominan ejercicios } \\
\text { numéricos. El contenido es ahistórico y descontextualizado. } \\
\text { El concepto cantidad de sustancia se desconoce. El } \\
\text { conocimiento histórico del concepto mol es erróneo o no se } \\
\text { aborda. }\end{array}$ \\
\hline & $\begin{array}{l}\text { Precuánticos y } \\
\text { cuánticos }\end{array}$ & $\begin{array}{l}3 \text { LTQ-EM (del periodo 1997-2003). Analiza el concepto } \\
\text { de discontinuidad de la materia (se aborda desde Dalton, } \\
\text { aunque se describen temáticas como distribución electrónica, } \\
\text { cambio químico, disoluciones y coloides sin aludir a la } \\
\text { cuántica). Hay referencia a las teorías atómicas, precuántica } \\
\text { y cuántica; se abordan las teorías atómicas desde los } \\
\text { griegos hasta la teoría atómica cuántica; se establecen los } \\
\text { conceptos de átomo, molécula y cambio químico; se hacen } \\
\text { representaciones de cada modelo atómico; sin embargo, } \\
\text { uno de ellos con errores conceptuales. }\end{array}$ \\
\hline
\end{tabular}

Fuente: elaboración propia. 
Los contenidos actitudinales se relacionan con las actitudes hacia la química y su aprendizaje.

Los contenidos procedimentales y actitudinales se encontraron en libros de texto más recientes: para los primeros, los estudios plantean que los LTQ se centran en resolver ejercicios numéricos para aplicar fórmulas o algoritmos; pocos LTQ consideran problemas cualitativos y abiertos o actividades que se complejicen. En los contenidos actitudinales, los análisis se centran en actitudes hacia la química asociados con la naturaleza de la ciencia y no de contenidos actitudinales hacia su aprendizaje (esto aún es incipiente), solo se da al incluir temas de interés para el alumnado.

\section{Subcategoría Relaciones metadisciplinares (RM) de los contenidos}

Los principales resultados de esta subcategoría se presentan en la tabla 3. Excepto dos trabajos, los demás enfatizan en la relación entre historia y epistemología (HE), sociología de la química, para entender la naturaleza propia de esta disciplina; o se analiza la inclusión de contenidos desde la relación química, tecnología y sociedad o enfoque CTS (Alba, 2014; Parga y Alba, 2015) -un solo trabajo, equivalente al $3 \%$ - y la inclusión de contenidos ambientales -un solo trabajo-.

Tabla 3. Subcategoría Relaciones de contenidos metadisciplinares (CM) de la química

\begin{tabular}{|c|c|}
\hline $\mathrm{CM}$ & Característica del análisis \\
\hline $\begin{array}{l}\text { Historia y } \\
\text { Epistemología }\end{array}$ & $\begin{array}{l}\text { Son estudios que analizan conceptos desde la historia de la química y su postura } \\
\text { epistemológica. Pocos estudios analizan teorías propias de los siglos xIx y xx, cuando } \\
\text { se dio la rivalidad entre teorías precuántica y cuántica; se presentan los experimentos } \\
\text { que permitieron la validación de modelos atómicos, pero no se contextualizan. } \\
\text { Aunque se alude al uso de teorías, no se hacen comparaciones, ni se explica por qué } \\
\text { una teoría superó a otra o como cambió. No se presentan preguntas o problemas } \\
\text { que fomentaron el avance en la química. No se refieren los textos originales (de } \\
\text { los científicos) ni las biografías de los científicos, esto hace que sean contenidos } \\
\text { descontextualizados, aproblemáticos y ahistóricos: hay descontextualización histórica } \\
\text { del contexto de producción de los modelos, se presenta el conocimiento químico } \\
\text { como algo concluido que prioriza la observación y el trabajo individual. } \\
\text { Los estudios resaltan que solo al comienzo de los textos hay interés por explicar qué } \\
\text { es la ciencia, cómo se trabaja, enfocada desde el "método científico" positivista, } \\
\text { aspectos que aluden a la naturaleza de la ciencia. Estas conclusiones concuerdan } \\
\text { con Farias (2012), quien afirma que en estos contenidos coexisten tres tipos de } \\
\text { relatos: el de la ciencia normativa, que fortalece la idea del método científico; el } \\
\text { de la histórica, que refleja la ciencia idealizada, y el de la ciencia real, que intenta } \\
\text { mostrar sin gran fuerza la ciencia de los científicos. }\end{array}$ \\
\hline Sociología & $\begin{array}{l}\text { Como categoría o criterio de análisis fue poco encontrado, sin embargo, en los que } \\
\text { se hizo el análisis se plantea que los LTQ no priorizan la ciencia como práctica; esto } \\
\text { no forma parte de estos materiales pues no se explica la química como ciencia ni } \\
\text { como actividad cultural. Cuando se enuncia la ciencia, se hace de forma general, al } \\
\text { comienzo de una unidad. }\end{array}$ \\
\hline Inclusión de CTS & $\begin{array}{l}\text { Se analizaron } 5 \text { LTQ-EM de los últimos cinco años. Se evidenciaron contenidos a } \\
\text { través de CTS, pero que aún no atienden lo deseable del enfoque (controversias } \\
\text { sociocientíficas e históricas, aplicaciones de la ciencia y la tecnología [CyT] de } \\
\text { forma interrelacionada; implicaciones políticas, económica, éticas y morales de } \\
\text { la CYT; alfabetizar en química, formación ciudadana desde la CyT; promoción de } \\
\text { nuevas visiones de la CyT, comprensión pública, entre otras). Los libros intentan } \\
\text { mostrar dichas relaciones cTS solo al incluir al inicio de unidad lecturas relacionadas } \\
\text { con el tema o como aplicaciones en un contexto (cotidiano o tecnológico) como } \\
\text { implementación de la química. }\end{array}$ \\
\hline
\end{tabular}




\begin{tabular}{|l|l|}
\hline \multicolumn{1}{|c|}{ CM } & \multicolumn{1}{|c|}{ Característica del análisis } \\
\hline $\begin{array}{l}\text { Inclusión de } \\
\text { contenidos } \\
\text { ambientales }\end{array}$ & $\begin{array}{l}\text { Se encontró un trabajo (Parga, 2017) que analizó } 4 \text { LT del periodo 2007-2012. En } \\
\text { este, los contenidos de química consideran aspectos ambientales de forma aislada } \\
\text { y con abordaje disciplinar, queriendo llamar la atención de un contenido disciplinar } \\
\text { determinado. Por ejemplo, para el contenido reacciones químicas se citan lecturas } \\
\text { o se contextualizan desde frases sobre el tema de contaminación del agua, del } \\
\text { aire, lluvia ácida, disminución de la capa de ozono, reciclaje, entre otros. Aún } \\
\text { falta comprensión o conocimiento de los principios de sustentabilidad ambiental } \\
\text { abordados como problema complejo, sistémico y multidisciplinar (y no un tema más) } \\
\text { en donde hay ausencia de lo social, económico, ecológico, ético, político y cultural. } \\
\text { Las actividades se proponen desde lo disciplinar y no retoman el problema ambiental } \\
\text { involucrado. Hay una ambientalización curricular en los LTQ incipiente. }\end{array}$ \\
\hline
\end{tabular}

Fuente: elaboración propia.

La relación He fue la predominante en los análisis (94\%), lo que viene sucediendo porque desde el ámbito internacional, a partir de las investigaciones en didáctica de las ciencias, se ha planteado como fundamental enseñar la química desde relaciones histórico epistemológicas (HE). Esto ha fomentado un buen número de trabajos que revisan, caracterizan y analizan los marcos conceptuales que adoptan los autores de LTQ, incluso los trabajos analizados llaman "transposición didáctica" o "confiabildiad de los libros", relaciones metadisciplinares, tramas histórico-epistemológicas, para determinar su correspondencia con lo que la comunidad científica ha construido de la química, y la perspectiva epistemológica, que evidencia cómo se ha ido construyendo la química como ciencia.

Este abordaje histórico-epistemológico (HE) de la química ha favorecido orientaciones en los libros de texto, que, según Farias (2012), han hecho que la mayoría de LTQ presenten episodios históricos que explican o justifican algunos aspectos de naturaleza de la ciencia, pero poco evidencian esta relación ya que lo HE se aborda de forma fragmentada y lo epistemológico está implícito en lo histórico. Se trate, en todo caso, de una historia anecdótica, centrada en personajes y sus principales aportes (como biografías), desconociendo la historia interna y externa. Sin embargo, los análisis evidencian LTQ que hacen abordajes contextualizados desde lo HE, algo de lo social, muy poco los principios CTS y ausencia de lo ambiental.

\section{Categoría 2: análisis didáctico de los contenidos}

Esta categoría emergió al identificar en los documentos investigaciones que analizan en los LTQ implicaciones didácticas, sus intenciones con respecto a la enseñanza y las formas de evaluar.

Esta categoría permitió establecer la forma de seleccionar, secuenciar y organizar contenidos. Se puso en evidencia que se hace desde la lógica disciplinar y no desde la lógica del pensamiento y los intereses del estudiantado, por lo que no facilitan la evolución a niveles que complejicen el conocimiento de los estudiantes. Se analizó si el diseño curricular es contextual y flexible, teorías y modelos que sustentan los aprendizajes, los referentes contextuales relacionados con a quién y dónde se enseña, así como las normas generales y del funcionamiento de las instituciones escolares a las que se dirigen los libros de texto (Carrión, 2014; Martínez y Téllez, 2012; Parga, Martínez y Téllez, 2013; 2012). Estos aspectos son importantes porque los docentes tienen orientaciones de las propuestas curriculares manifiestas en los LTQ, que contienen 
implícitos o explícitos, modelos pedagógico-didácticos, posturas epistemológicas y, por tanto, visiones de la química.

\section{Subcategoría Implicaciones didácticas (ID)}

En esta se consideran criterios del modelo didáctico explicitado por los LTQ y suS implicaciones. Esto es fundamental, pues como afirma Medina y Sanmartín (1990) parece que se ha perdido confianza en los libros de texto porque los principios didácticos (por ejemplo del constructivismo, del enfoque CTS, enseñanza para la comprensión o $\mathrm{EpC}$, contenidos contextualizados, resolución de problemas, cuestiones controversiales, entre otros) nose abordan y el profesorado prefiere materiales con contenido tradicional, supeditando lo conceptuala otros criterios de enseñanza.

Así, se encontraron trabajos que identificaron, en la mayoría de LTQ, metodologías superficiales, que no exigen esfuerzo a los estudiantes, porque las actividades son de aplicación de algoritmos (resultados mecánicos) y reproducción de conceptos o teorías presentados. El conocimiento químico se organiza desde definiciones que deben memorizarse más que comprenderse o aplicarse en la resolución de ejercicios, más que problemas contextuales. Se encontraron actividades para el "desarrollo de competencias", pero, por la manera en la que se diseñan, no atienden a este objtetivo, no hay un progreso en las capacidades y destrezas del estudiantado. Esto evidencia falta de coherencia entre principios, enfoques didácticos y contenidos desarrollados (conceptuales, procedimentales, actitudinales), por lo que la mayoría de estudios concluye que predomina el modelo tradicional de enseñanza.

\section{Subcategoría Intenciones de la Enseñanza (IE)}

Los estudios que lo analizan plantean que:

- La mayoría de LTQ se declaran constructivistas, y asumen indagar ideas previas con respecto a conceptos; estas actividades se presentan solo al comienzo de una unidad; no se cuestiona lo que va pasando con la evolución conceptual del alumnado. Algunas de las actividades de ideas previas evalúan información de memoria.

- Las intenciones de enseñanza están lejos de proponer complejizar el conocimiento del alumnado.

- Si bien los contenidos y principios del enfoque CTs favorecen la comprensión del funcionamiento de la ciencia, la tecnología y su desarrollo, los LTQ poco explicitan dentro de sus intenciones estas comprensiones, ni cómo se pueden favorecer frente a perspectivas filosóficas, sociológicas, políticas, históricas y epistemológicas de lo enseñado. 
- Faltan integraciones de las intenciones didácticas de los LTQ con los contenidos, las actividades y las secciones, para tener unidad y no fragmentaciones.

- Los contenidos se organizan de forma tradicional en la secuencia establecida por los textos (que no se articula con la lógica del pensamiento del estudiantado), no se organizan progresiones, para establecer relaciones entre contenidos conceptuales, procedimentales y actitudinales; no es evidente la conexión entre un tema y otro, lo que desfavorece la comprensión de la química.

- Se fomenta el trabajo colectivo e individual en actividades experimentales, lo cual es favorable, pero en el desarrollo conceptual esta imagen de construcción del conocimiento (colectivo) no se evidencia ni se destaca.

- Varios de los contenidos se presentan en contextos cotidianos del estudiantado (con ejemplos de la vida diaria en los que un fenómeno está presente) y al indagar algunas ideas previas; otros contextos no se explicitan ni implicitan (HE, social, disciplinar), por ello, no es intención de la enseñanza el establecerlos para resolver problemas en los que la química esté involucrada.

\section{Subcategoría Formas de evaluar (FE)}

- En general, la evaluación que proponen los LTQ se analiza poco en las investigaciones. Los trabajos que lo hacen, enfatizan en las actividades presentes, que se clasifican por tipo de competencia por "desarrollar" o idea previa a evaluar, consulta a realizar, o ejercicio algorítmico y problema por resolver.

- Las actividades se presentan como secciones exclusivas (actividades de inicio, desarrollo y final de unidad), actividades de laboratorio, pequeños proyectos; lecturas de comprensión de fenómenos; varios de los análisis consideran que las actividades (sobre todo las de lápiz y papel) pueden tener poco sentido para los estudiantes por centrarse en ejercicios algorítmicos y repetir un modelo abordado en el LTQ; estas no se proponen para fomentar la participación en la toma de decisiones o para complejizar niveles de comprensión.

- No se evidencia una evaluación continuada en los libros de texto y la evaluación se reduce a la presentación de actividades que reproducen lo mencionado en el libro.

- Por el tipo de actividades propuestas, poco se puede estar mejorando la actitud hacia el aprendizaje de la química, lo que favorece la llamada "quimifobia".

\section{Categoría 3: Evolución (E) de los LTQ}

Esta categoría emergió al identificar en los documentos investigaciones que analizan la evolución de los LTQ. Así, se encontró un estudio que analizó seis LTQ para determinar sus características desde los años sesenta hasta hoy (Carrión, 2014). El análisis fue contextual con respecto a la época en que fueron publicados. Como unidades de análisis se crearon categorías como Naturaleza de la ciencia, Contenidos y Modelo didáctico; así, se determinó en qué aspectos han evolucionado estos materiales. El estudio evidenció 
evolución respecto a la naturaleza de la ciencia y al modelo didáctico, pero no es una evolución altamente deseable, pues los LTQ deberían estar acordes a una enseñanza de la química para el siglo XXI.

Con respecto a los contenidos, no se evidencia gran evolución, es decir, no se tienen en cuenta mecanismos e instrumentos para la construcción del conocimiento químico; se siguen enseñando los mismos contenidos de forma descontextualizada, aunque desde los años noventa se involucran aspectos cotidianos del estudiantado, hecho que se articula con lo He de la química que está ausente en la mayoría de LTQ. Sobre la concepción de ciencia, esta ha ido evolucionando lentamente, de visiones ingenuas, empiristas, positivas a una más racional moderna en muy pocos casos, pero que aún hoy no muestra cómo se cambia una teoría por otra, por ejemplo. Por tanto, es una visión mixturada y no nítida dentro de una tipología de ciencia moderna y menos aún posmoderna. En cuanto al modelo didáctico, los libros de las primeras décadas no se preocupaban por explicitarlo (aunque se centraban en informar y transmitir contenidos disciplinares); los de las últimas décadas sí, pero aún falta coherencia entre los principios de cada modelo y lo que desarrollan. Se ha ido pasando de modelos de transmisión de información a modelos de descubrimiento y con esbozos de un constructivismo, pero aún incipiente.

\section{Categoría 4: los autores}

Esta categoría no se identifica como emergente en los documentos analizados, pero se plantea porque los investigadores en didáctica de las ciencias se han preocupado por los libros de texto, los estudiantes, el profesorado y el currículo, pero siguen desconociendo la influencia que en los libros de texto tienen las editoriales comerciales y sus autores, ya que una ciencia escolar diferente, una que se acerque más a lo que es la actividad científica (Farias, 2012) requiere una nueva visión que se refleje en libros de texto acordes a las problemática y exigencias del hoy. En este sentido, esta categoría se crea al analizar conocimientos/creencias metadisciplinares, pedagógico-didácticas y químicas, latentes en los documentos Sin embargo, ninguno de los trabajos analizados estudió de forma explícita esta característica, excepto Villa (2008), que evaluó la trayectoria académica de los autores llamando la atención sobre su ausencia en la mayoría de LTQ. Así que desde las categorías de la tabla 1, se dedujo la categoría Autores desde los hallazgos obtenidos en el presente estudio:

- Frente a los conocimientos/creencias de la química de los autores, los documentos analizados plantean la presencia de errores conceptuales, desconocimiento de las versiones actuales de conceptos, teorías y modelos de la química pues en el intento de hacer enseñable un contenido, se termina distorsionado el conocimiento químico. 
- En cuanto a los conocimientos/ creencias de los autores sobre las metadisciplinas de la química (su historia, epistemología, sociología, tecnología), en la mayoría predominan visiones estereotipadas de la ciencia, positivistas (ejercicios para demostrar, laboratorios para comprobar, experimentos para reproducir, teorías para comprobar, observaciones que hacer, etc.); muy pocos proponen actividades basadas en problemas por resolver, que develen una visión de ciencia y de su construcción diferente para esta nueva época; fueron pocas las relaciones de la química con otros campos, se asumen solo para lecturas de inicio o fin de capítulos como formas de contextualizar lo que se enseña, las interrelaciones con problemas ambientales, sociales, políticos económicos de la química no se asumen.

- Conocimientos/creencias de los autores frente a lo pedagógico-didáctico: si bien los libros más actuales tienden a asumir modelos constructivistas, no es coherente cuando la enseñanza se centra en contenidos lineales, desarticulados, descontextualizados (los clásicos contenidos); actividades que no proponen niveles de evolución, que son abundantes como queriendo decir "entre más ejercicios, más se aprende", que preparan para pruebas de Estado, complementados con secciones de lecturas de relaciones CTS, de proyectos, pero que se ven como añadidos y no abordajes integrales en las unidades. Existe, por tanto, una mixtura de conocimientos/ creencias que funde lo tradicional con pinceladas de propuestas pedagógico-didácticas más actuales.

Considerando las categorías emergentes en este análisis documental y las recomendaciones de los investigadores consultados, se proponen algunas mejoras en los LTQ:

- Presentar la química como una disciplina que ayude a tomar conciencia de los problemas y de las situaciones de interés local/mundial desde los cuales pueda aportar. La tendencia curricular es que estas problemáticas definen los contenidos y metodologías por enseñar, no al contrario. Tener contenidos sin saber para qué o qué sentido tienen es hoy cuestionable.

- Usar imágenes, gráficos, esquemas, etc., con criterios contextuales (didácticos, disciplinares, metadisciplinares), no solo con fines estéticos.

- Elaborar unidades didácticas evolutivas desde tramas didácticas acerca de las principales teorías de la química que contribuyan a las problemáticas de hoy.

- LOs LTQ comerciales son materiales que se deben usar de manera crítica, reflexiva, como parte del modelo didáctico del profesor, como apoyo de la planeación curricular, no deben definir ni el contenido ni las intenciones de enseñanza. Por esto, se sugiere contar con libros de texto contextualizados, hechos por los mismos docentes.

- Abordar aspectos de género (la mujer en la ciencia), la ciencia en la sociedad y la cultura. 


\section{Conclusiones}

El presente artículo mostró resultados de un estudio cualitativo documental de las investigaciones hechas en Colombia sobre los libros de texto de química, a partir del análisis de tres tipos de documentos: trabajos de grado, disertaciones de maestría y artículos publicados sobre investigaciones de LTQ. Se destacan:

- En los 33 documentos analizados, la principal tendencia se relaciona con investigaciones centradas en conceptos, y su desarrollo sobre aspectos histórico-epistemológicos; esto definió la primera categoría del análisis. En este sentido, la mayoría de propuestas editoriales desconocen la historia-epistemología al abordar un concepto, y lo presentan descontextualizados tanto en el aspecto histórico como en el social, el epistemológico e incluso el disciplinar de la química. Esto concuerda con lo planteado por Malaver et al. (2004) y con González-Canle y Sánchez-Gómez (2014), que plantean que cuando se aborda, sobre todo lo histórico, se hace en términos anecdóticos, con visiones simplistas y reducidas, que llevan a una imagen de ciencia producto de trabajos aislados, en los que no ha habido comunidad, disputas, intercambios, consensos, y desligados de teorías y de modelos teóricos.

- Llama la atención que las mismas investigaciones analizadas se enfocaran en conceptos sin ser parte de teorías y de los modelos. Fueron pocos los estudios que analizaron teorías propias de la química (dual-estructural, flogisto-oxígeno, equivalentismo-atomismo) o de la física que son fundamentales para la química (precuánticos-cuánticos). En este sentido, se concluye que las propuestas de LTQ desconocen tales teorías y los criterios metadisciplinares que las rodean.

- Fueron pocas las investigaciones que analizaron aspectos didácticos de los LTQ (categoría 2), su evolución (categoría 3) y a los autores de estos materiales (categoría 4). La categoría 2 evidencia que las propuestas didácticas están desarticuladas de modelos pedagógico-didácticos; unas son las intenciones y otros los desarrollos: hay énfasis en caracterizar ideas previas al comienzo de cada unidad -pero como actividad que evalúa con un solo instrumento, cuando esto se hace con y desde diversos instrumentos y a lo largo de la enseñanza-; hay proyectos de final de capítulo, cuando podrían ser desde el comienzo para articular un gran proyecto para desarrollar-; en evaluar desde competencias con actividades de inicio y de final que no evidencian evolución de los aprendizajes; en proponer lecturas complementarias como mecanismo para generar interés del estudiante hacia el aprendizaje de la química, pero que pueden generar la idea de una química aplicada a..., en preparar para las pruebas de Estado (en el caso de LTQ-EM). 
Así, hay una tendencia de un modelo transmisionista, que va hacia un descubrimiento dirigido, pasando por el de descubrimiento autónomo, pero incipiente desde un posible constructivismo (categoría 3). Es decir, al ser libros de texto escritos en los últimos cuarenta años, falta mucho para que los más recientes sean propuestas acordes a lo que proponen las investigaciones en didáctica de la química con respecto a las exigencias sociales y las problemáticas que hoy nos atañen.

- Finalmente, no hubo estudios enfocados en los autores de LTQ; sus conocimientos/creencias frente a la química, sus metadisciplinas, sus concepciones pedagógico-didácticas; solo un trabajo consideró la ausencia de la formación o currículo de los autores para garantizar la idoneidad del profesional que escribe estos materiales.

- Dos de las tres categorías caracterizadas en el presente estudio coinciden con dos de las categorías internacionales: la de contenidos propios de la química (aunque aludiendo a conceptos, teorías, modelos -tales como el modelo atómico de Rutherford-, enlace químico, electroquímica, isómeros, modelo del octeto de Lewis y Langmuir, modelos moleculares de Kossel, Newton Lewis y Linus Pauling, mol y cantidad de sustancia, teoría estructural en química orgánica, hipótesis de Avogadro, modelo semicuántico de Bohr, teorías del oxígeno y del flogisto, proteínas combustión, $\mathrm{pH}$, discontinuidad de la materia, principio de Le Chatelier y equilibrio químico, aromaticidad, hibridación, reacciones químicas, fenómeno químico y valencia), y las de los estudios relacionados con el uso de la historia-epistemología al abordar conceptos particulares. Solo se encontró un trabajo sobre el papel de las ilustraciones, por tanto no se asumió como categoría para Colombia, aunque exista en el contexto internacional.

- Es necesario recomendar a las editoriales y sus autores superar las deficiencias de los LT, que si bien han venido cambiando, lo han hecho con lentitud. Entre tanto, al profesorado le imponen -las instituciones educativas, en la mayoría de los casos- trabajar con libros que no puede seleccionar por su calidad; por ello es necesario insistir en formar al profesorado para que sea autor de los diseños curriculares que usará en el aula: es decir, para que diseñe según sus conocimientos/creencias, contextos y particularidades propias del alumnado y la escuela.

\section{Referencias}

Denzin, N. y Lincoln, Y. (2012). Manual de investigación cualitativa volumen II. Paradigmas y perspectivas en disputa. Barcelona: Gedisa.

Farias, D. (2012). Teoría, estructura y modelos atómicos en los libros de texto de química de educación secundaria. Análisis desde la sociología de la ciencia e implicaciones didácticas (tesis). Universidad de Barcelona, España.

Galeano, M. (2012). Estrategias de investigación social cualitativa: el giro en la mirada. Medellín: La Carreta.

González-Canle F. y Sánchez-Gómez, P. (2014). Contenidos de estructura atómica y molecular 
en libros de texto españoles de química general (1928-1978). Enseñanza de las Ciencias, 32(3), 671-689.

López-Valentín, D. M. y Guerra-Ramos, M. T. (2013). Análisis de las actividades de aprendizaje incluidas en libros de texto de ciencias naturales para educación primaria utilizados en México. Enseñanza de las Ciencias, 31 (2), 173-191.

Malaver, M., Pujol, R. y d'Alessandro, M.A. (2004). Los estilos de prosa y el enfoque ciencia-tecnología-sociedad en textos universitarios de química general. Enseñanza de las ciencias, 22(3), $441-454$.

Medina, M. y Sanmartín, J. (1990). Ciencia, tecnología y sociedad. País Vasco: Anthropos Editorial del Hombre.

Ministerio de Educación Nacional, MEN (2004). Estándares básicos de competencias en ciencia naturales y ciencias sociales. Serie Guía 7. Bogotá: Cargraphics.

Mora, W. y Parga, D. (2008). El conocimiento didáctico del contenido en química: integración de las tramas de contenido histórico-epistemológicas con las tramas de contexto-aprendizaje. Tecné, Episteme y Didaxis, TED, 24, 56-81.

Occeli, M. y Valeiras, N. (2013). Los libros de texto de ciencias como objeto de investigación: una revisión bibliográfica. Enseñanza de las Ciencias, 31 (2), 133-152.

Parga, D. L. (ed.) (2015). Conocimiento didáctico del contenido (CDC) en química. Bogotá: Universidad Pedagógica Nacional.

Perales, F. y Vílchez, J. M. (2015). Iniciación a la investigación educativa con estudiantes de secundaria: el papel de las ilustraciones en los libros de texto de ciencias. Enseñanza de las Ciencias, 33(1), 243-262.

Ruiz, J. (2009). Metodología de la investigación cualitativa. Bilbao: Universidad de Deusto.

Valles, M. (2009). Técnicas cualitativas de investigación social. Reflexión metodológica y práctica profesional. Madrid: Síntesis Sociología.

\section{Para citar este artículo}

Parga, D. L. (2018). Investigaciones en Colombia sobre libros de texto de química: análisis documental. Tecné, Episteme y Didaxis: TED, 44, 111-128. 


\section{Anexo 1}

Documentos analizados

\begin{tabular}{|c|c|}
\hline Tipo de documento & Referencia \\
\hline $\begin{array}{l}\text { Disertación de maestría } \\
\text { (DM) en la UPN }\end{array}$ & 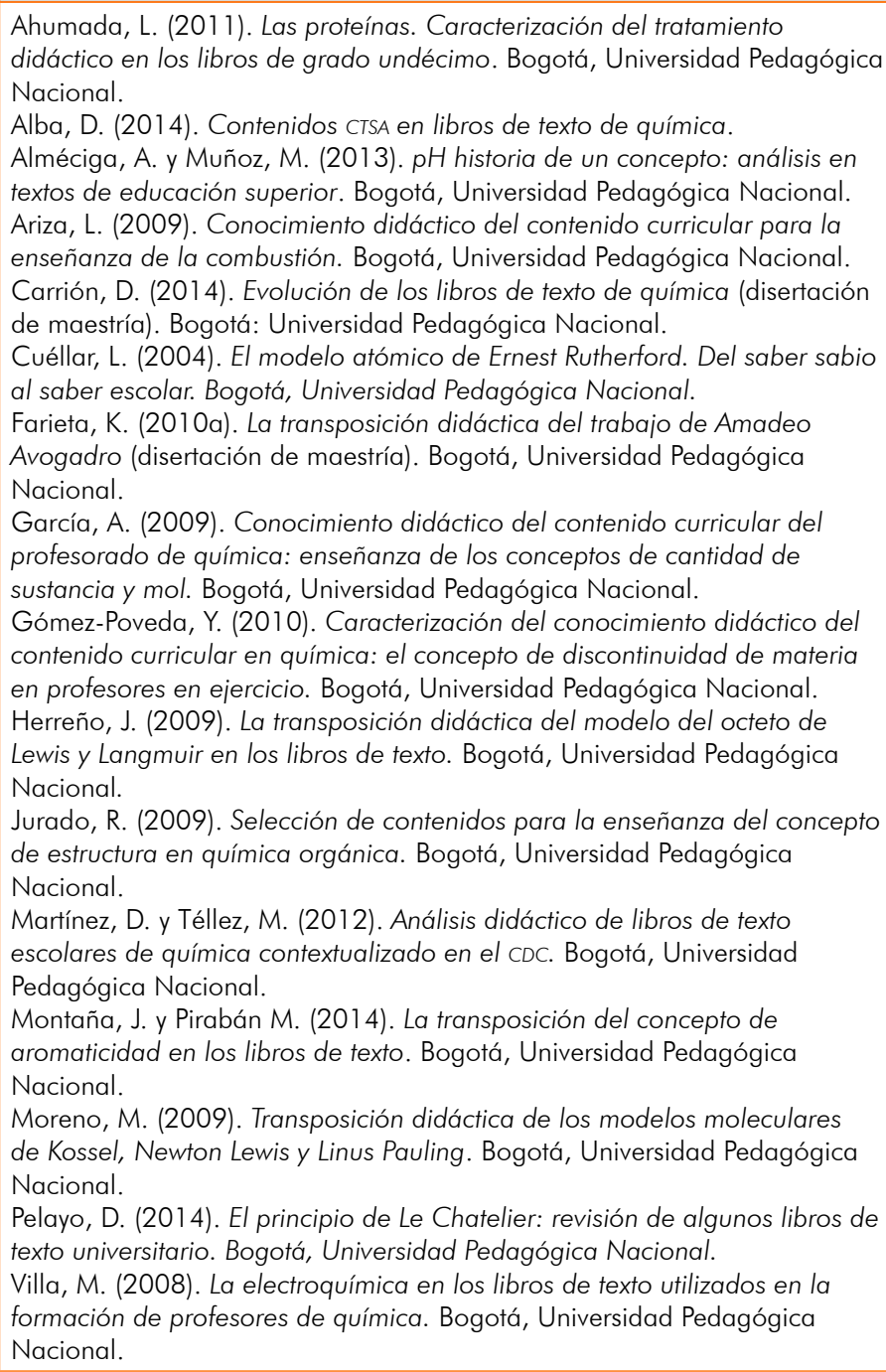 \\
\hline
\end{tabular}




\begin{tabular}{|c|c|}
\hline Tipo de documento & Referencia \\
\hline $\begin{array}{l}\text { Artículo/ponencia } \\
\text { publicados } \\
\text { (AP) }\end{array}$ & 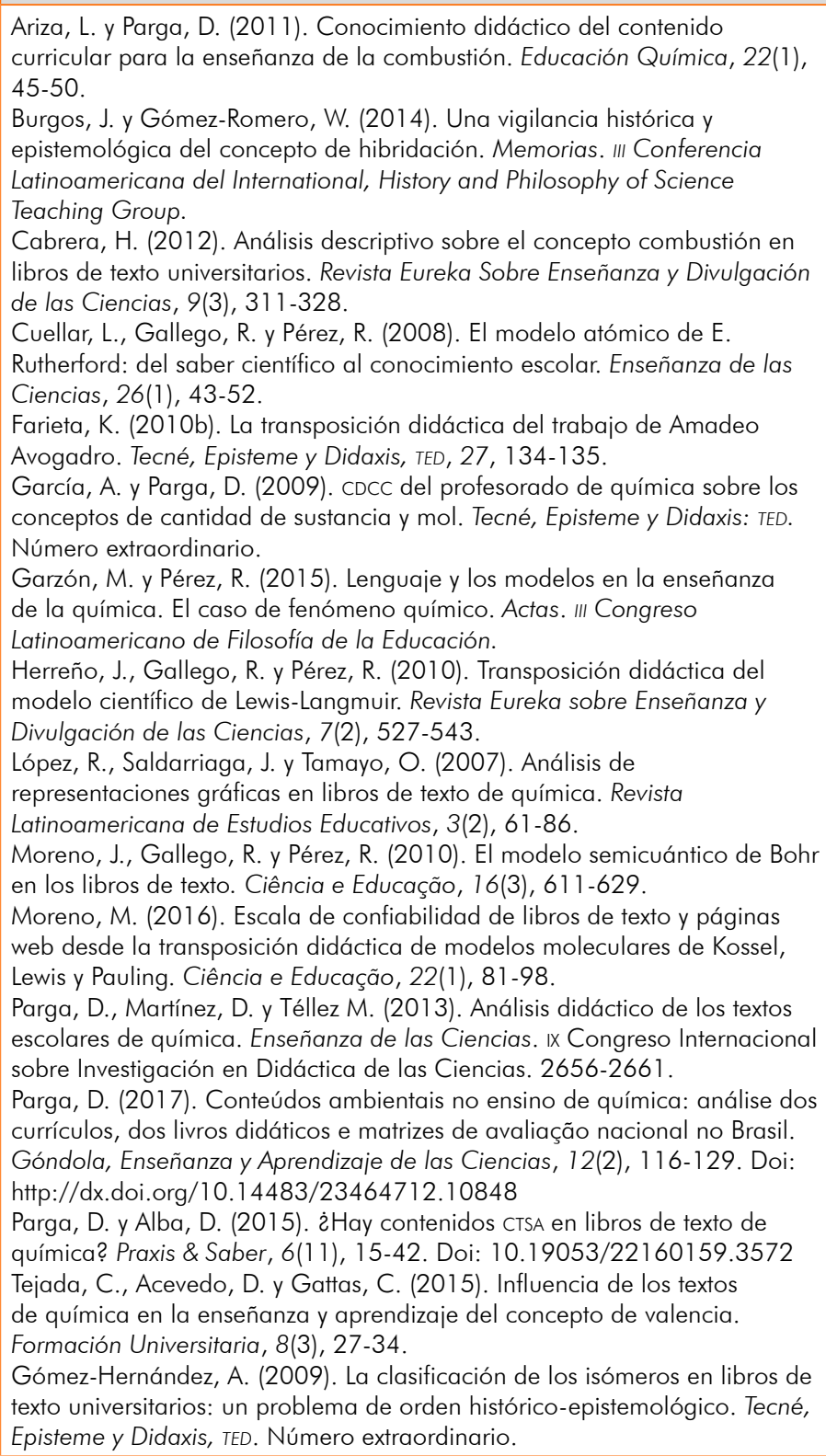 \\
\hline Trabajo de grado (TG) & $\begin{array}{l}\text { Gutiérrez, O. y Reyes, H. (2014). Las reacciones químicas: un análisis de la } \\
\text { retórica en los libros de texto universitarios. Cali, Universidad del Valle. }\end{array}$ \\
\hline
\end{tabular}

